\title{
Generation of a superposition of odd photon number states for quantum information networks
}

\author{
J. S. Neergaard-Nielsen ${ }^{(1,3)}$, B. Melholt Nielsen ${ }^{(1,3)}$, C. Hettich ${ }^{(1,3)}$, K. Mølmer ${ }^{(2,3)}$, and E. S. Polzik ${ }^{(1,3)}$ \\ ${ }^{1}$ Niels Bohr Institute, Copenhagen University, DK 2100, Denmark \\ 2 Department of Physics and Astronomy, University of Aarhus, DK 8000, Denmark \\ ${ }^{3}$ QUANTOP, Danish Research Foundation Center for Quantum Optics
}

\begin{abstract}
We report on the experimental observation of quantum-network-compatible light described by a non-positive Wigner function. The state is generated by photon subtraction from a squeezed vacuum state produced by a continuous wave optical parametric amplifier. Ideally, the state is a coherent superposition of odd photon number states, closely resembling a superposition of weak coherent states (a Schrödinger cat), with the leading contribution from a single photon state in the low parametric gain limit. Light is generated with about 10,000 and more events per second in a nearly perfect spatial mode with a Fourier-limited frequency bandwidth which matches well atomic quantum memory requirements. The generated state of light is an excellent input state for testing quantum memories, quantum repeaters and linear optics quantum computers.
\end{abstract}

PACS numbers: 03.65.Wj; 03.67.-a; 42.50.Dv

Generation of non-classical, non-Gaussian states of light compatible with atomic quantum memory has been an outstanding challenge driven by various applications in quantum information processing. Storage of single photon states in atomic memories is at the heart of proposals for quantum repeaters [1], whereas Fock states or Schrödinger cat states, stored off-line, form the basis of linear optics quantum computing [2, 3]. Various approaches to generation of single photon states compatible with atoms have been pursued: single atoms in free space [4] and in high-finesse cavities [5, 6], and atomic ensembles [7, [8, 9], and non-classical features such as photon antibunching and violation of classical inequalities have been demonstrated. However, the dominant contribution to the detected state of light in all of those experiments has been a vacuum state, due to either high losses or high mode impurity. High fidelity single photon states [10, 11] have been reported previously but those were not compatible with atomic memories. Schrödinger cat states have been demonstrated for microwave radiation 12. but not for light.

In this Letter we report on generation of light, which is compatible with atomic quantum memories and which is characterized by negative values of the Wigner function. The generated states are close to cat states with small coherent amplitude, and converge to a single photon state for certain experimental parameters. The compatibility with quantum memories is ensured by the purity of the spatial, temporal modes of the field. The generated field has a well defined Fourier-limited spectrum with a bandwidth around $9 \mathrm{MHz}$ in the low gain regime, is frequency tunable, and has a perfect spatial Gaussian profile.

We follow the essence of the proposal 13] in subtracting a photon from squeezed vacuum by transmitting squeezed light through a weakly reflecting beam splitter and detecting a photon in the reflected beam. In 14] this idea was used for demonstration of generation of non-Gaussian states. In the absence of losses, the state 13 in case of, e.g., $3 \mathrm{~dB}$ of squeezed input is
$\Psi \sim|1\rangle+0.29|3\rangle+0.09|5\rangle+\ldots$ in the Fock basis. It has been shown in 13, 15, 16] that such a state well approximates a small-amplitude Schrödinger cat state.

In order to generate a narrow-band state of light in a pure spatial mode, we make use of a continuous wave $(\mathrm{cw})$ optical parametric oscillator (OPO) below threshold for generation of the initial squeezed field. Such a device has been successfully used for generation of squeezed and entangled Gaussian states compatible with atomic targets 17, 18, 19, 20, 21]. In an OPO the non-linear optical medium is placed inside an optical resonator (Fig. 1), thus providing light generated within well defined frequency and spatial modes. At the same time the resonator significantly enhances the spectral brightness of the selected modes. All those features prove advantageous for the OPO-based source compared to single-pass sources [10, 11, 14] as far as compatibility with atomic memories is concerned. The cw character of the experiment requires a multimode theory for its adequate description. We have developed this theory based on the formalism of Gaussian states and Gaussian covariance matrices 22] (see also an alternative theoretical multimode analysis for a different detection model [23]).

In the experiment (Fig. 11) the output of the Ti:Sapphire laser around $852 \mathrm{~nm}$ (close to the Cesium D2 transition) is frequency doubled to generate up to $150 \mathrm{~mW}$ of the blue OPO pump beam $(426 \mathrm{~nm})$, while a small part of the laser beam is split off to act as the local oscillator (LO). The OPO is an $81 \mathrm{~cm}$ long bow-tie cavity with a $10 \mathrm{~mm}$, temperature-tuned $\mathrm{KNbO}_{3}$ crystal. The down-conversion is Type I with $90^{\circ}$ non-critical phase matching, hence all down-converted photon pairs are generated in the same polarization and in the same direction defined by the spatial mode of the resonator. The OPO cavity is locked on resonance with the laser frequency, so that the degenerate down-conversion mode is efficiently generated. The output coupling mirror of the OPO has a transmission of $12.7 \%$ and the intracavity losses are $1.0 \%$. In addition to this, the blue pump 


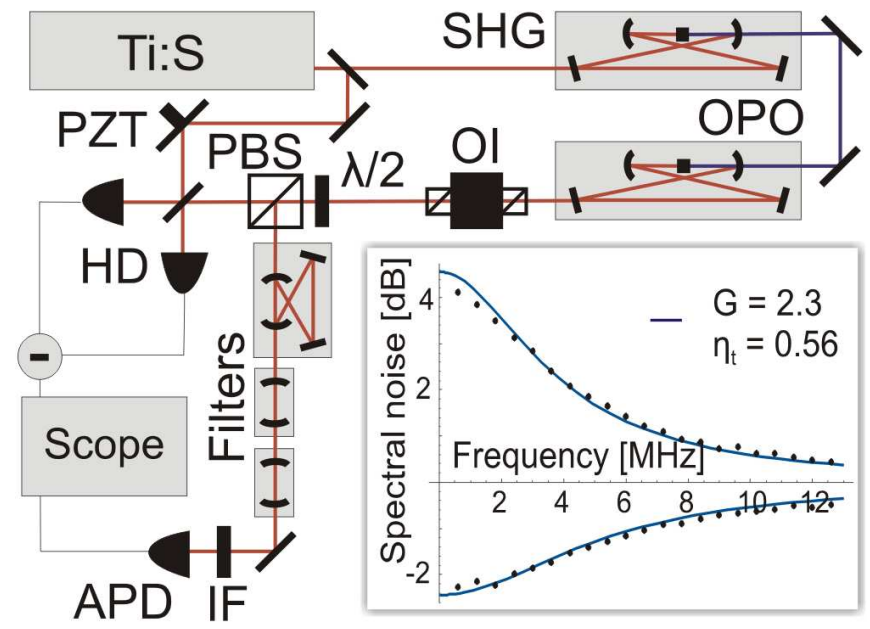

FIG. 1: (Color online) Experimental setup. SHG - second harmonic generator, OI - optical isolator (to prevent scattered LO light from entering the OPO), $\lambda / 2$ - half wave plate, PBS - "magic" beam splitter, PZT - piezo mounted mirror, HD homodyne detector, IF - interference filter, APD - avalanche photo diode. The inset shows the squeezing spectrum obtained from a Fourier analysis of the raw data. The blue curve is the best fit of the theoretical spectrum. The fitted parameters (OPO gain 2.3, and total efficiency, $\eta_{t}=0.56$ ) match well the experimentally estimated values.

induces around $1 \%$ absorption of the IR cavity field due to the light-induced loss process in the crystal - BLIIRA 24]. Altogether, this gives a cavity bandwidth of 4.4 $\mathrm{MHz} \mathrm{HWHM}$ and the output coupling efficiency of the cavity around $\eta_{O P O}=0.86$. More details of this setup can be found in [17, 18, 20].

In order to characterize squeezing generated by the OPO and the losses in the homodyne channel, we measure the squeezing spectrum of the OPO. The OPO output is mixed on a balanced $50 / 50$ beamsplitter with a few-milliwatt LO beam. The differential photocurrent is measured by two low noise photodetectors and its Fourier transform is digitally obtained. The results are presented in the inset in Fig. 1. Note that squeezing is observed across the entire theoretical bandwidth of the OPO (with the exception of frequencies below $30 \mathrm{kHz}$ ), and not just within a narrow frequency band. This is necessary for the photon subtracted state generation. From this data we infer the OPO gain and the overall losses. The overall propagation efficiency 0.66 coincides reasonably well with $\eta=\eta_{O P O} \eta_{p r}\left(\eta_{h o m}\right)^{2}$ calculated from the measured values for $\eta_{O P O}$, propagation efficiency from the OPO to detectors $\eta_{p r}=0.88$ and homodyne efficiency $\eta_{\text {hom }}=0.96$. Our homodyne detectors have the quantum efficiency of 0.90 which along with the small dark current lead to the effective detector efficiency $\eta_{d}=0.85$, giving the total efficiency of detection $\eta_{t}=0.60$ (close to $\eta_{t}=0.56$ inferred from squeezing data).

For the photon subtraction experiment the downconverted output of the OPO is directed onto a "magic" beam splitter (polarizing beam splitter cube in combination with a half-wave plate) which reflects a small portion $(3-7 \%)$ of the light towards a photon counter - APD (Perkin-Elmer SPCM-AQR-13, dark count rate $160 \mathrm{~s}^{-1}$ ). Note that for low reflectivity of the "magic" beam splitter the probability of a two-photon click is very low, i.e., a click of the APD almost for sure corresponds to the single photon detection. Besides the degenerate squeezed mode the OPO generates thousands of, non-degenerate, modes which are also phase matched and resonant with the cavity. Photons in those modes produce unwanted counts in the photon subtraction channel, and therefore have to be filtered out. Towards this end, on the way to the APD, the light is filtered with three consecutive cavities and a spectral filter which block all the OPO output modes except for the degenerate squeezed mode (Fig. 1). The OPO cavity and the three filter cavities are tuned in resonance with the degenerate OPO mode by injecting a weak coherent seed beam into the OPO. The count rate of the APD is then used to produce the error signal for the frequency locking. A LabView program monitors this count rate while it scans all cavities at different low frequency rates until the program finds a setting for the four piezo control voltages which gives an expected maximal count rate. Under stable lab conditions the lock is reliable down to a count rate of approximately 5000 $\mathrm{s}^{-1}$. With the cavities lock points established, we inject the blue pump into the OPO and turn down the coherent seed beam keeping the lock operational now with only the down-converted light. By turning the "magic" beamsplitter to full reflection, we measure the maximum count rate as a reference for the subsequent setting of the

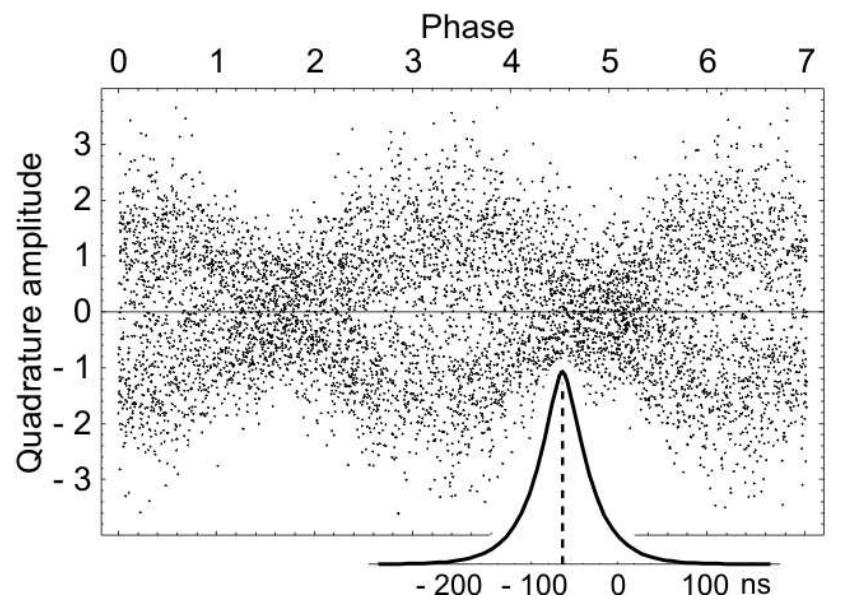

FIG. 2: Part of a typical trace obtained after application of the temporal mode function (shown in the inset) to each of the 20,000 raw data segments. Each segment of raw data is multiplied by the mode function and integrated, producing one phase/quadrature point for the trace. The phase of the LO has been scanned at roughly $1 \pi / \mathrm{s}$. The temporal mode function is shifted with respect to the APD click due to the delay in electronics. 


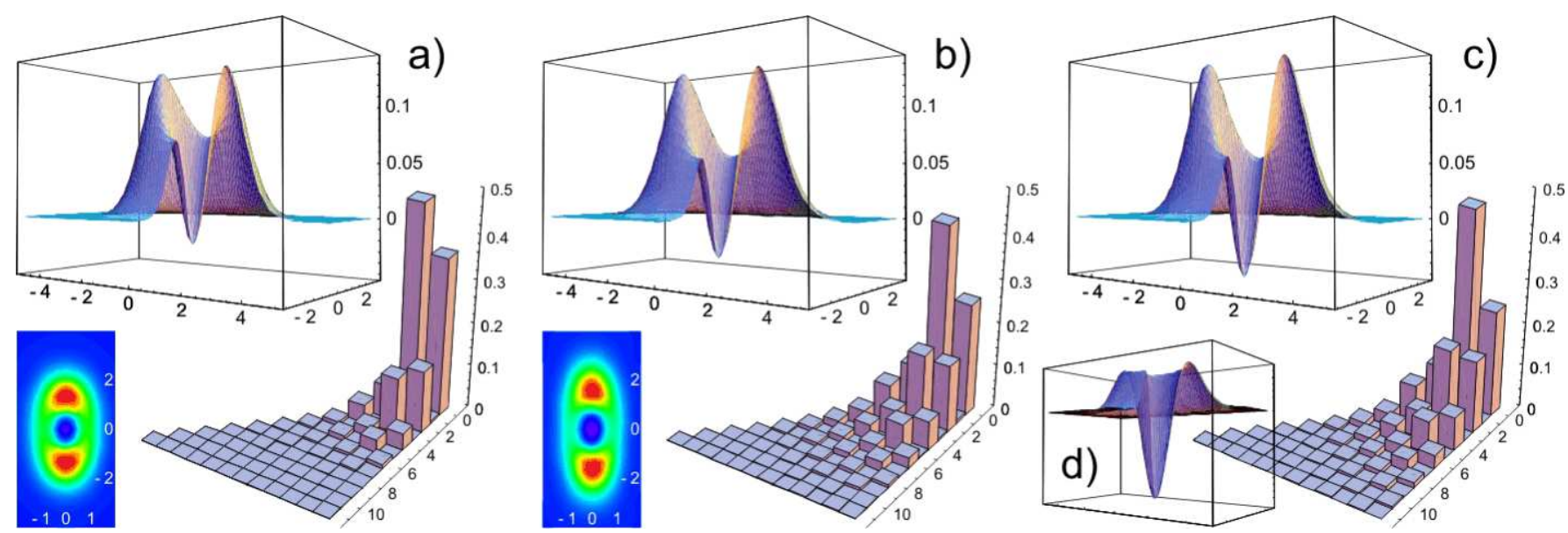

FIG. 3: (Color online) Figures a,b) show the density matrices and Wigner functions (including a top-down contour plot) reconstructed from experimental data. a) the OPO gain 1.8 b) gain 2.3. The presented plots are averages of 50 separate states, each reconstructed from a 20,000 points quadrature trace as explained in the text and corrected for the $85 \%$ detector efficiency. c) state calculated from full multi-mode theory with the same temporal mode function and the same overall propagation efficiency $\eta=0.64$ as for the experimental state b). d) state calculated with perfect efficiency $\eta=1$.

beamsplitter at the chosen reflection ratio. At the same time we get an estimate for the total production rate of photons into the degenerate mode (around $2 * 10^{6} \mathrm{~s}^{-1}$ for the parametric gain 2.3).

The LO phase is scanned to perform the tomography of the state. For every click of the APD, a data segment of 1000 points is recorded within a period starting $1 \mu \mathrm{s}$ before and ending $1 \mu \mathrm{s}$ after the trigger event. 20,000 such segments are stored and comprise one full measurement of the different quadratures of the quantum state. The total acquisition time of a full measurement is only around 3 seconds and a new measurement can be initiated a few seconds later. The high spectral brightness of our source thus allows faster data acquisition than in single pass experiments where it may take hours.

During the initial data processing each $2 \mu$ s segment of experimental data is turned into a single quadrature measurement using a particular temporal mode function. If the $\mathrm{OPO}$ is in the intermediate to high gain regime, the field populates many modes, and the optimum mode function should be chosen as the one populated by a quantum state with the strongest non-classical features, e.g., the one with the largest negative value attained by the Wigner function. A fairly simple Ansatz for the mode function $\kappa e^{-2 \pi \gamma|t|}-\gamma e^{-2 \pi \kappa|t|}$, which is the Fourier transform of the product of two Lorentzians with half-widths $2 \pi \gamma$ and $2 \pi \kappa$, shows close to the optimal performance. The optimal $\gamma$ and $\kappa$ are close to $2 \pi * 9 \mathrm{MHz}$ (OPO width) and $2 \pi * 48 \mathrm{MHz}$ (filter cavity width). The temporal shape of the generated state is shown in Fig. 2 Every $2 \mu \mathrm{s} / 1000$ points data segment is then multiplied by the mode function. This gives a number which is the measured quadrature amplitude value for that segment. This procedure is equivalent to the use of a pulsed local oscillator with the temporal shape of the mode func- tion. A full measurement set consists of 20,000 quadrature values, along with their corresponding phase values (obtained from the time of their acquisition via an independent phase calibration). A data set obtained in this way is presented in Fig. 2] The lack of points around zero values of the quadrature phase amplitudes is a clear signature of the non-Gaussian character of the radiation. We perform the same procedure for the vacuum state, and use the result for normalization.

To reconstruct the density matrix from the data we use the iterative maximum-likelihood method of 25], based on [26] - for reviews of this and other methods, see [27]. In order to obtain the Wigner function of the optical field irrespectively of the detector inefficiency we correct the density matrix for the detector efficiency using the algorithm of 25]. The density matrices and the Wigner functions of the photon subtracted squeezed vacuum states for two different degrees of squeezing are shown in Fig. Ba,b. Each presented state is an average of 50 individually acquired 20,000-point measurements. The averaged values of the Wigner functions at the origin are, respectively, $W(0,0)=-0.028 \pm 0.010$ and $W(0,0)=-0.040 \pm 0.014$ (we note that even the states reconstructed without correction for detector efficiency have small negative dips). The negative regions of the Wigner functions show their profoundly non-classical character. The Wigner functions are elongated along the direction of the parent squeezed states signifying the presence of three photon and five photon contributions. In the limit of very low degree of squeezing the elongated shape would be reduced to a round shape characteristic for the single photon state. Fig. 31 shows the state calculated following our theoretical model with the efficiency $\eta=0.64$ corresponding to the experimental efficiency of the state shown in Fig. 3 $\mathrm{b}$. This number is a product of 
$\eta=0.66$ obtained from the squeezing data and an extra factor 0.97 which partially accounts for dark counts of the APD. As seen from Fig. 3. and 3r, theory and experiment are in good agreement. The small discrepancy may be attributed to an admixture of other modes in the trigger channel.

Due to the cw character of the squeezing produced in the experiment, the single mode theory of [13, 15, 16, 28] is not fully applicable. We have developed an exact theoretical treatment, which is possible because the output from the OPO is a Gaussian state fully described by its second order moments, i.e., by the two-time correlation functions of the output $\left\langle\hat{b}^{\dagger}(t) \hat{b}\left(t^{\prime}\right)\right\rangle$ and $\left\langle\hat{b}(t) \hat{b}\left(t^{\prime}\right)\right\rangle$ [29]. If the transmission (reflection) of the "magic" beam splitter is $|\tau|^{2}\left(|\rho|^{2}=1-|\tau|^{2}\right)$ and if the filters for the trigger field can be modeled by a single Lorentzian of half width $2 \pi \kappa$, the annihilation operator for the mode, causing the click of the APD at $t=t_{c}$ can be expressed as $\hat{a}_{t_{c}}=2 \rho \sqrt{\pi \kappa} \int_{-\infty}^{t_{c}} \exp \left(-2 \pi \kappa\left(t_{c}-t\right)\right) \hat{b}(t) d t+\hat{F}_{\text {noise }}$ where $\hat{F}_{\text {noise }}$ represents vacuum field contributions from the "magic" beam splitter and from the frequency filters, and its precise form does not need to be specified. For an arbitrary mode function $u(t)$, we obtain a similar expression for the annihilation operator of the field mode extracted by the corresponding LO detection within the time mode $u(t), \hat{a}_{u}=\tau \int_{-\infty}^{\infty} u(t) \hat{b}(t) d t+\hat{G}_{\text {noise}}$, where $\hat{G}_{\text {noise }}$ is a vacuum noise term. Losses and detector efficiencies are incorporated by reduction factors on the first terms and additional vacuum noise contributions in the equations for $\hat{a}_{t_{c}}$ and $\hat{a}_{u}$. The second moments of the four field quadrature variables for these operators are directly obtained from the output correlation functions and the filter and mode functions. Defining the $4 \times 4$ covariance matrix $\gamma$ by its elements $\gamma_{i j}=2 \operatorname{Re}\left\langle y_{i} y_{j}\right\rangle$, with the four Hermitian field quadratures forming a column vector $\mathbf{y}=\left(y_{1}, y_{2}, y_{3}, y_{4}\right)^{T}$, the Wigner function can be written explicitly, $W(\mathbf{y})=1 /(\pi \sqrt{\operatorname{det}(\gamma)}) \exp \left(-\mathbf{y}^{T} \gamma^{-1} \mathbf{y}\right)$. This Gaussian Wigner function fully characterizes the quantum state of the trigger and LO modes prior to the APD detection event. The action of a photo detection event is described by application of the corresponding field annihilation operator to the quantum state, which in the
Wigner function representation is represented by a combination of differentiation and multiplication by the $y_{i}$ arguments [29]. The reduced state associated with the homodyned field mode is subsequently obtained by tracing over the trigger field state, i.e., by integrating over the trigger mode quadrature arguments in $W$. These operations can be done explicitly, and the resulting single mode phase space function is readily obtained (Fig. 31). For further discussions of this treatment, see [22].

The state generated by photon subtraction from single mode squeezed vacuum is a superposition of odd-number Fock states [13], which overlaps with a cat state $|\alpha\rangle-|-\alpha\rangle$ with better than $99 \%$ fidelity for coherent amplitudes less than $\alpha=1.2$. In the present $c w$ experiment we have several modes populated, hence we are bound to get contributions from even number states, and e.g. the vacuum, which grow with the degree of squeezing. The states shown in Fig. 3a,b overlap with cat states with coherent amplitudes $\alpha=1.05$ and $\alpha=1.3$ with a fidelity of $53 \%$. For our squeezing, the overlap between the lossless state (Fig. 35) and a cat state would be still quite good, 0.95 and 0.90 , respectively. A major improvement of the purity of cat states can be achieved by reducing optical losses. It seems feasible to obtain an OPO escape efficiency $\eta_{O P O}=0.95$, a propagation efficiency $\eta_{p r}=0.97$ and a homodyne efficiency $\eta_{\text {hom }}=0.99$, thus reaching a value close to 0.87 for the total efficiency. Even higher purity can be obtained with purification procedures proposed in [15, 28].

In conclusion we have demonstrated experimentally a new type of non-classical state of light, the photon subtracted squeezed vacuum state based on an optical parametric oscillator below threshold. The results are in good agreement with a complete multi-mode theoretical analysis. With the time mode function of $100 \mathrm{nsec}$, and the transform limited bandwidth around $10 \mathrm{MHz}$, frequency tunable around Cesium D2 transition, the source is compatible with atomic memories and networks.

After submission of this paper, generation of an optical "kitten" state was reported by Ourjoumtsev et al. [30].

This research has been funded by EU grants COVAQIAL and QAP.
[1] H.J. Briegel et al, Phys. Rev. Lett. 81, 5932 (1998).

[2] E. Knill et al, Nature 409, 46 (2001).

[3] T.C. Ralph et al, Phys. Rev. A 68, 042319 (2003).

[4] Darquie B et al, Science 309 (5733), 454-456 (2005).

[5] J. McKeever et al, Science 303, 1992-1994 (2004).

[6] A. Kuhn et al, Phys. Rev. Lett. 89, 067901 (2002).

[7] C.W. Chou et al, Phys. Rev. Lett. 92, 213601 (2004).

[8] T. Chaneliere et al, Nature 438, 833-836 (2005).

[9] M.D. Eisaman et al, Nature 438, 837-841 (2005).

[10] A.I. Lvovsky et al, Phys. Rev. Lett. 87, 050402 (2001).

[11] A. Zavatta et al, Science 306, 660-662 (2004).

[12] G. Nogues et al, Phys. Rev. A 62, 054101 (2000).
[13] M. Dakna et al, Phys. Rev. A 55, 3184 (1997).

[14] J. Wenger et al, Phys. Rev. Lett. 92, 153601 (2004).

[15] A.P. Lund et al, Phys. Rev. A 70, 020101(R) (2004).

[16] M.S. Kim et al, Phys. Rev. A 71, 043805 (2005).

[17] E.S. Polzik et al, Phys. Rev. Lett. 68, 3020,(1992).

[18] N.Ph. Georgiades et al, Phys. Rev. Lett. 75, 3426, (1995).

[19] J. Hald et al, Phys. Rev. Lett. 83, 1319(1999)

[20] C. Schori et al, Phys. Rev. A 66, 033802 (2002).

[21] C. Schori et al, Phys. Rev. Lett. 89, 057903 (2002).

[22] K. Mølmer, to appear in Phys. Rev. A.

[23] M. Sasaki and S. Suzuki, Phys. Rev. A 73, 043807 (2006).

[24] H. Mabuchi et al, JOSA B 11, 2023 (1994). 
[25] A.I. Lvovsky, J. Opt. B: Quantum Semiclass. Opt. 6, S556-S559,(2004).

[26] Z. Hradil, Phys. Rev. A 55, R1561 (1997); K. Banaszek, ibid. 57, 5013 (1998); K. Banaszek et al, ibid. 61, 010304 (1999); Řeháček et al, ibid. 63, 040303 (2001).

[27] G.M. D'Ariano et al, quant-ph/0507078 A.I. Lvovsky and M.G. Raymer, quant-ph/0511044

[28] H. Jeong and T.C. Ralph, quant-ph/0509137

[29] C.W. Gardiner, Quantum Noise, Springer-Verlag, Berlin 1991.

[30] A. Ourjoumtsev et al, Science 312, 83-86 (2006). 